\title{
Fabrication and Characterization of Arrays of GaAs All-Optical Logic Gates
}

\author{
M. Warren ${ }^{1}$, Y.H. Lee ${ }^{1}$, G.R. Olbright ${ }^{1}$, B.P. McGinnis ${ }^{1}$, H.M. Gibbs ${ }^{1}$, \\ N. Peyghambarian ${ }^{1}$, T. Venkatesan ${ }^{2}$, B. Wilkens ${ }^{2}$, J. Smith ${ }^{3}$, \\ and $A$. Yariv ${ }^{3}$ \\ 1 Optical Sciences Center, University of Arizona, Tucson, AZ 85721, USA \\ 2 Bell Communications Research, Murray Hill, NJ 07974, USA \\ 3 California Institute of Technology, Pasadena, CA 91125, USA
}

Optical bistable elements fabricated from gallium arsenide (GaAs) have demonstrated potential as optical logic elements for use in optical parallel processing systems [1]. In previous experiments, the devices have consisted of small, uniform slabs of active material, either bulk GaAs or GaAs-AlGaAs multiple-quantum-well structures [2]. These were placed between mirrors to form nonlinear Fabry-Perot etalons. The active regions of these earlier devices were grown with top and bottom "windows" of AlGaAs to reduce the exciton surface-recombination rate. The overall switching time of these devices was limited by the carrier relaxation time to several nanoseconds. The present devices were produced from a $1.5-\mu m-t h i c k$ GaAs layer, grown by molecular beam epitaxy $(M B E)$ on a single-crystal GaAs substrate with an underlying $0.2-\mu \mathrm{m}-$ thick $\mathrm{Al}_{0.4} \mathrm{Ga}_{0.6} \mathrm{As}$ etch stop layer, but no top window. The absence of the window resulted in an order-of-magnitude reduction in the recovery time [3]. Some of the wafer was etched to form arrays of small GaAs mesas or "pixels" on a single substrate. The etched surface structure served to further reduce the recovery time, as well as producing an array of separated devices on a single substrate [4]. Each individual pixel of the array measured $9 \mu \mathrm{m} \times 9 \mu \mathrm{m}$, and the pixels were spaced $20 \mu \mathrm{m}$ apart from each other (Fig. 1). Individual samples of the array, as mounted in etalons, typically contained several thousands of pixels. However, the fabrication process used for these devices $c$ an be easily scaled to larger sizes. Large arrays of optically bistable elements may become important components of powerful optical parallel processing systems. Such processors could make efficient use of the inherent parallelism of optics.

To produce the array samples, pieces of the wafer were coated with photoresist. The pieces were then contact-printed with a custom-designed photomask and developed to produce an array of photoresist squares on the GaAs surface. The wafer was then etched in a reactive-ion-etch system with a Freon $12\left(\mathrm{CCl}_{2} \mathrm{~F}_{2}\right)$, helium and oxygen mixture [4,5]. The selective etch process stopped at the AlGaAs layer, resulting in an array of sharply defined pixels. The photoresist pattern was then removed with an oxygen plasma in the reactive-ion-etch system. The GaAs substrate layer of all the samples was removed, first by grinding the sample thin, and then by a selective wet-chemical etch [6].

Several methods of mounting the samples between mirrors to form etalons were tried. The devices used for bistable and logic-gate operation were glued between dielectric-coated glass microscope coverslips. Some etalons were formed by direct deposition of the dielectric coatings on both sides of the GaAs arrays. The spectral transmission of the latter etalons was measured at various points to obtain some measure of the uniformity of the GaAs material. Regions containing a few tens of etched pixels had most of the pixels with transmission peaks within one instrument width of each other. Larger areas exceeded this spread because the sample thickness was wedged, probably resulting from the removal of the substrate. 


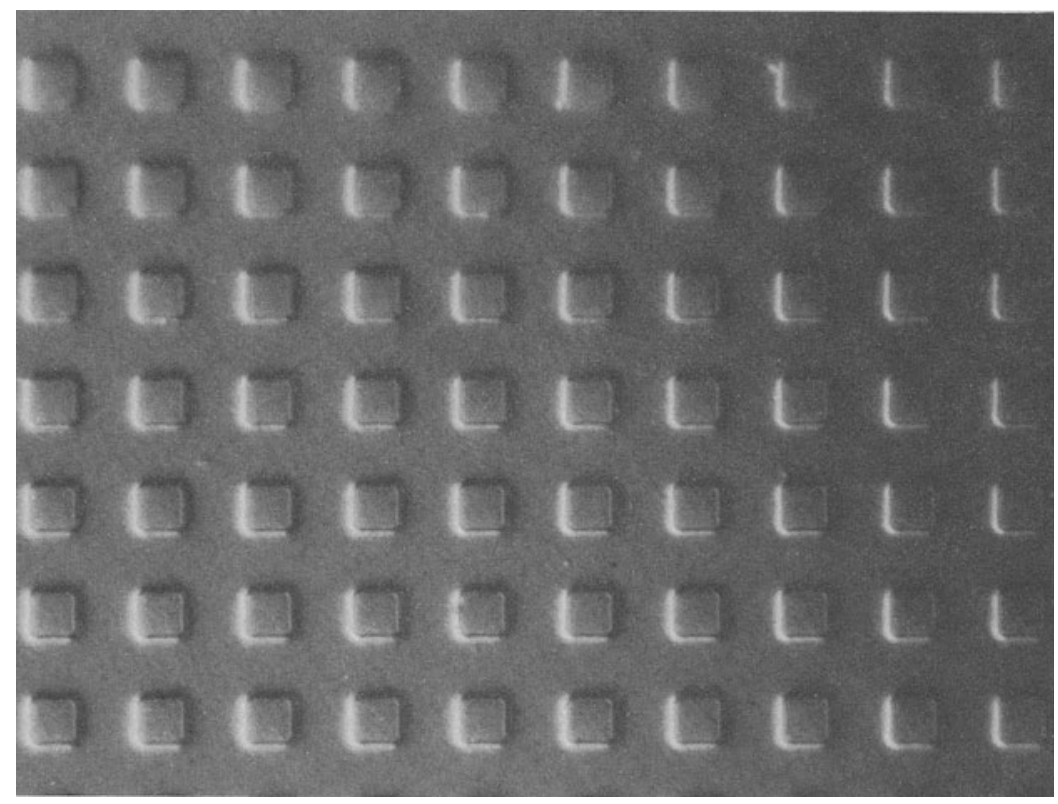

Fig. 1. Photomicrograph of 2-D array of 9- $\mu \mathrm{m} \times 9-\mu \mathrm{m}$ pixels prepared by reactive-ion etching.
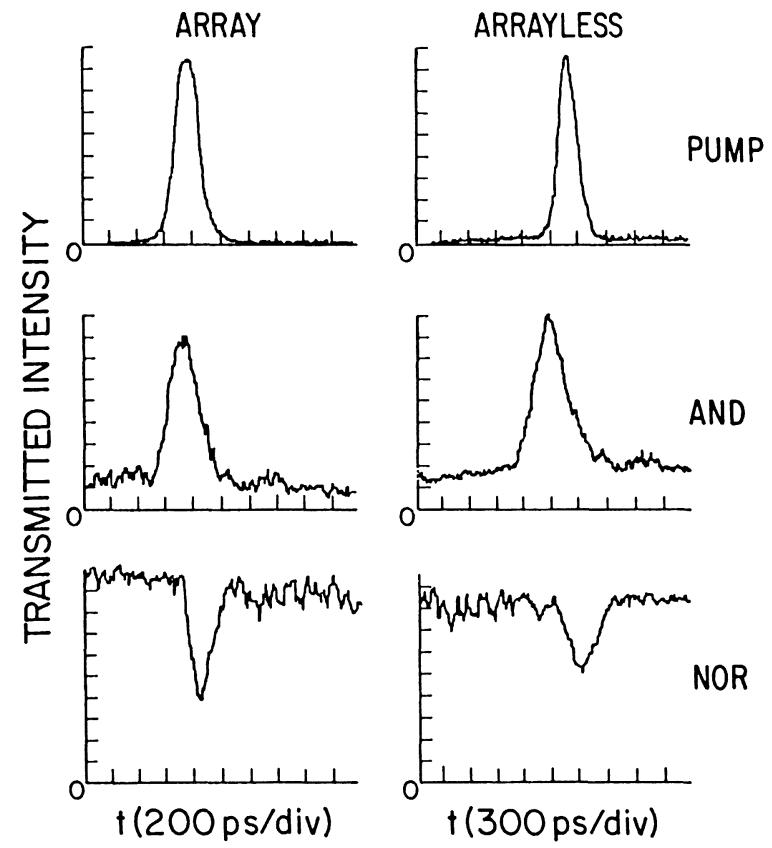

Fig. 2. Streak camera traces of the temporal evolution of the input pump pulse and the $\mathrm{cw}$ probe transmission for AND-gate and NOR-gate operation using one $9-\mu \mathrm{m} \times 9-\mu \mathrm{m}$ pixel of the windowless GaAs etalon. The base lines are taken with the probe beam blocked. 
An array etalon and an unpatterned etalon were operated as NOR and AND gates in a pump-and-probe experiment. The pump pulses were of 180-ps duration with $1-n J$ energy at $5145 \AA$. The probe pulses were tuned below the bandgap, and their transmission was monitored by a streak camera [3].

For a single pixel of the array, a gate recovery time of less than 200 ps was observed. The arrayless sample, with the same 10- $\mu \mathrm{m}$ spot size, showed a gate recovery time of 300 ps (see Fig. 2). The enhanced surface recombination rate apparently accounts for the short relaxation times in the devices. The faster recovery time in the array compared with the arrayless device may be due to the shorter average carrier diffusion distance to a surface and to possible etch damage [4].

Both the array and unpatterned devices have been operated as bistable devices. The devices were cooled to $150 \mathrm{~K}$ to be compatible with the tuning range of a Nd:YAG pumped dye laser. Operation was with 10-ns pulses and relatively low peak power values of $5 \mathrm{~mW}$. Switch-off times were around $1 \mathrm{~ns}$ [7].

In summary, GaAs bistable devices have been fabricated in large arrays. By removal of the customary top AlGaAs window, fast logic-gate operation has been demonstrated. These results show promise for the fabrication of arrays of optical gates capable of handling parallel logic operations at high data rates.

\section{Acknow ledgment}

The Arizona portion of this research was supported by the Optical Circuitry Cooperative, Air Force Office of Scientific Research, Army Research Office, and National Science Foundation.

\section{References}

[1] J. L. Jewe11, Y. H. Lee, M. Warren, H. M. Gibbs, N. Peyghambarian, A. C. Gossard, and W. Wiegmann, Appl. Phys. Lett. 46, 918 (1985).

[2] N. Peyghambarian and H. M. Gibbs, Opt. Eng. 24, 68 (1985).

[3] Y. H. Lee, M. Warren, G. Olbright, H. M. Gibbs, N. Peyghambarian, T. Venkatesan, J.S. Smith, and A. Yariv, "Streak-camera observation of 200-ps recovery of an optical gate in a windowless GaAs etalon array," submitted to Appl. Phys. Lett.

[4] T. Venkatesan, B. Wilkens, M. Warren, Y. Lee, G. Olbright, H. M. Gibbs, N. Peyghambarian, J. S. Smith, and A. Yariv, "Fabrication of arrays of GaAs optical bistable devices," submitted to Appl. Phys. Lett.

[5] K. Hikosaka, T. Mimura, and K. Joshin, Jpn. J. Appl. Phys. 20, L847 (1981).

[6] J. L. Jewe11, H. M. Gibbs, A. C. Gossard, A. Passner, and W. Wiegmann, Materials Letters 1, 148 (1983).

[7] M. Warren et al., to be published. 